\title{
NEW PHOTOPOLYMER WITH TRIFUNCTIONAL MONOMER FOR HOLOGRAPHIC APPLICATIONS
}

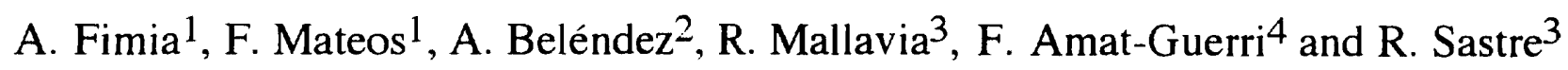

(1) Laboratorio de Optica. Departamento Interuniversitario de Optica Universidad de Alicante. Apdo. No. 99. E-03080 Alicante, Spain

(2) Departamento de Ingeniería de Sistemas y Comunicaciones

Universidad de Alicante. Apdo. No. 99. E-03080 Alicante, Spain

(3) Instituto de Ciencia y Tecnología de Polímeros

CSIC, Juan de la Cierva 3, E- 28006 Madrid, Spain

(4) Instituto de Química Orgánica General

CSIC, Juan de la Cierva 3, E- 28006 Madrid, Spain

$$
\begin{array}{ll}
\text { Phone: } & +34-6-5903509 \\
\text { Fax: } & +34-6-5903464 \\
\text { e-mail: } & \text { fimia@vm.cpd.ua.es }
\end{array}
$$

Part of this paper was presented at the IS\&T/SPIE Symposium in Electronic Imaging, Science and Technology (Holographic Materials), February 5-10, San Jose, California (USA).

Applied Physics B: Lasers and Optics 


\begin{abstract}
New holographic recording materials based on photopolymerizable systems have contributed significantly to the recent growth of holographic applications. Previously; we reported that in photopolymerizable systems with a difunctional monomer, ethylene glycol dimethacrylate (EGDMA) improves the behaviour of the system and explains the role played by an eosin ester that has an oxooxime group in the production of amine initiator radicals. This comparative study was carried out in our laboratory using differential scanning photocalorimetry and holography. The results of the new photosensitive recording materials for holography indicate that this system can be used for the formulation of very promising photopolymers that have better performance. The aim of this study was to change the crosslinking monomer in order to increase the energetic sensitivity and discover the rest of the behavior. The new photopolymerizable mixture containing pentaethritol triacrylate (PETA), in a $1: 1$ ratio of volume and a 2-hydroxyethylene methacrylate monomer (HEMA). A diffraction efficiency of $80 \%$ is achieved with an energetic sensitivity of $3 \mathrm{~J} / \mathrm{cm}^{2}$ at $514 \mathrm{~nm}$., and the spatial resolution is up to $2000 \mathrm{l} / \mathrm{mm}$.
\end{abstract}

PACS: $42.40,81.20 . \mathrm{Sh}$ 


\section{1. - INTRODUCTION}

Recording materials for Holography range from photographic emulsions to thermoplastics and lithium niobate cristals, depending on the type of hologram required. Thermoplastics are excellent real-time materials for holographic interferometry, are reusable and produce good quality images. Dichromated gelatins are used for manufacturing holographic optical elements with high diffraction efficiencies, excellent signal to noise ratio and high enviromental stability.

The optimization of all types of recording materials is connected to their application, and we can say that the knowledge we have gained about their photochemical processing has progressed hand in hand with the knowledge about their optimization. One of the most important aspects of a holographic material is its energetic sensitivity in the area of the spectrum in which the sensitizer used in its composition acts.

Photopolymers can be considered holographic materials because of their many attractive features and these materials have potential applications in high-density storage and optical data processing $[1,2]$. Holographic photopolymers basically consist of one monomer or a mixture of monomers, and a photoinitiator. Acrylamide has been one of the most frequently used monomers due to the fact it can be polymerized. In addition, the sensitizers that have been developed make it possible to work in virtually all zones of the electromagnetic spectrum in which visible lasers exist. Previous studies have produced a new sensitizer that permits an increase in energetic sensitivity [3], in comparison with comercial dyes. However, the system of monomers used in previous experiments continues to have a low degree of sensitivity. In this study we incorporate a new composition into the system of monomers in order to increase photopolymerization speed by increasing energetic sensitivity while at the same time maintaining other important properties of the holographic recording material such as its response to changes in spatial frecuency and its diffraction efficiency.

The optimization process was carried out under the assumption that it is possible to increase reaction speed by means of copolymerization processes in which a combination of bifunctional and trifunctional monomers which increase reaction speed, while 


\section{2.- EXPERIMENTAL RESULTS}

The experiments were carried out using a holographic set-up in which two collimated beams were made to incide on the sample, in a symmetric geometry, the sample was placed between two $10 \times 10 \mathrm{~cm}$, sheets of glass with spacers that guaranteed a thickness of $45 \pm 5 \mu \mathrm{m}$. The wavelength used was $514 \mathrm{~nm}$, from an Ar laser. In Table I we present the base composition of the system to which we have added a trifunctional component (PETA) in the same concentration as the EGDMA monomer to optimize the polimeric composition.

The formation of the hologram can be observed according to the set-up represented in Figure 1. In this method of detection of the diffracted light, a chopper is used in the object beam. When the chopper blocks the object beam, only the reference wave strikes the sample. The light of this wavelength is deflected in the direction of the object wave as the hologram grows and can be detected with a photodiode which is connected to a boxcar integrator, therefore we measured the diffracted light only when the object beam was blocked so that the hologram would grow. In this way the Bragg relation is automatically satisfied and changes in thickness are not influences to measurements of diffraction efficiencies [4].

The first step in optimization was to change the component called EGDMA for PETA and change the recording spatial frequency while keeping the storage intensity constant.

Figure 2 shows the response of the diffraction efficiency as a function of the recording energy for compositions including EGDMA and PETA for different spatial frecuencies. A summary of these results is presented in Figure 3, which shows a bit of a decrease in the diffraction efficiency when the spatial frecuency is increased for compositions with PETA. As regards these results we should remember that the trifunctional groups have greater molecular size than the bifunctional ones. This makes a 
slight decrease in diffraction efficiency possible when the storage spatial frecuency increases. However, as Figure 4 shows, the energy needed to reach maximun diffraction efficiency is the same for different spatial frequencies for PETA but not for EGDMA, as the latter requires four times more energy at low spatial frequencies and seven times more at high spatial frequencies.

The seconds optimization parameter is the intensity of the light beams during storage if we consider that these materials have clearly non-linear behavior [5].

As we can see in Figure 5, the response of the PETA when analyzing the energy needed to reach maximum diffraction efficiency as a function of the intensity required to store the holographic grating is clearly lineal. However, as Figure 5 shows, the response of the EGDMA is non-linear. If we do a study of the diffraction efficiency as a function of storage intensity, we find that the behavior of both systems is similar even though the PETA system reminds us a great deal of the conversion curves of a typical polymerization reaction, as we can see in Figure 6 . This result is extremely important given that it establishes the correlation between the conversion curves that are used in photocalorimetry and the diffraction efficiency curves used in holography to optimize photochemical systems [3].

We can also see that the behaviour of the diffraction efficiency response curves for systems that have bifunctional and trifunctional monomers corresponds to the conversion behavior that these types of monomers have when they are analyzed by photocalorimetry. As can be seen in Figures 5 and 6, both monomers have the same response to a change in the recording beam intensity, although EGDMA comes closer to reaching maximum diffraction efficiency at a lower intensity. When we optimized the storage intensity $\left(24 \mathrm{~mW} / \mathrm{cm}^{2}\right)$, we found that both components allow us to obtain diffraction efficiencies higher than $60 \%$, even though PETA achieves maximum diffraction efficiency at an energy of $3 \mathrm{~J} / \mathrm{cm}^{2}$ as compared to the $16 \mathrm{~J} / \mathrm{cm}^{2}$ that EGDMA requires. In Figure 7 we show diffraction efficiency as a function of exposure when storage intensity remains constant $\left(24 \mathrm{~mW} / \mathrm{cm}^{2}\right)$ and we can cearly see the different energy needed to reach maximum diffraction efficiency. 


\section{4.- CONCLUSIONS}

The correct selection of the composition and utilization of trifunctional monomer like PETA make it possible to increase the sensitivity of photochemical systems that work through photopolymerization. Once again it has been shown that these systems are non-linear behavior in relation to storage intensity. This is due to the non-linear behavior of the copolymerization reactions that are produced during the illumination of the sample as well as the possible diffusion phenomena that have recently been published as regards this type of photopolymerization materials [6]. By using these systems, diffraction efficiencies of almost $80 \%$ can be achieved when energy is $3 \mathrm{~J} / \mathrm{cm}^{2}$.

\section{ACKNOWLEDGMENTS}

We thank the Comisión Interministerial de Ciencia y Tecnologia (CICYT) of Spain for financial support (Projects MAT93-0369 and MAT93-0757) 


\section{REFERENCES}

1.- R. A. Lessard: Proc. SPIE, 2405, 2 (1995)

2.- U. Rhee, H. J. Caulfield, J. Shamir, C. S. Vikram and M. M. Mirsalehi: Opt. Eng., 32, 1839 (1993)

3.- R. Mallavia, F. Amat-Guerri, A. Fimia and R. Sastre: Macromolecules, 27, 2643 (1994)

4.- A. Beléndez, I. Pascual and A. Fimia: J. Opt. Soc. Am.-A, 9, 1214 (1992)

5.- A. Fimia, F. Mateos, R. Sastre, J. Pineda and F. Amat-Guerri: J. Mod. Opt., 41, $1867(1994)$

6.- G. Zhao and P. Mouroulis: J. Mod. Opt., 41, 1929 (1994) 


\section{FIGURE CAPTIONS}

Figure 1.- A schematic representation of the set-up for the recording of holographic growth curves.

Figure 2.- Diffraction efficiency as a function of exposure energy for different spatial frequency.

Figure 3.- Diffraction efficiency as a function of spatial frecuency for EGDMA and PETA monomers.

Figure 4.- Energy for maximun diffraction efficiency as a function of spatial frecuency.

Figure 5.- Energy for maximun diffraction efficiency as a function of recording intensity.

Figure 6.- Diffraction efficiency as a function of recording intensity.

Figure 7.- Diffraction efficiency as a function of energy for PETA and EGDMA when the storage intensity remains constant $\left(24 \mathrm{~mW} / \mathrm{cm}^{2}\right)$. Spatial frequency 950 lines $/ \mathrm{mm}$.

\section{TABLES}

Table I.- The base composition of the photopolymer system. 
TABLE I
$\mathrm{N}$-methyldiethanolamine (MDEA)
$3.9 \times 10^{-2} \mathrm{M}$
2-hydroxiethyl methacrylate (HEMA)
$3.88 \mathrm{M}$
Photoinitiator
$1.9 \times 10^{-3} \mathrm{M}$

Ethylene glycol dimethacrylate (EGDMA)

Pentaerythritol triacrylate (PETA)

The monomer ratio (HEMA):
$2.58 \mathrm{M}$

$2.58 \mathrm{M}$

crosslinking agent (PETA o EGDMA)

is $1: 1$ in volume 


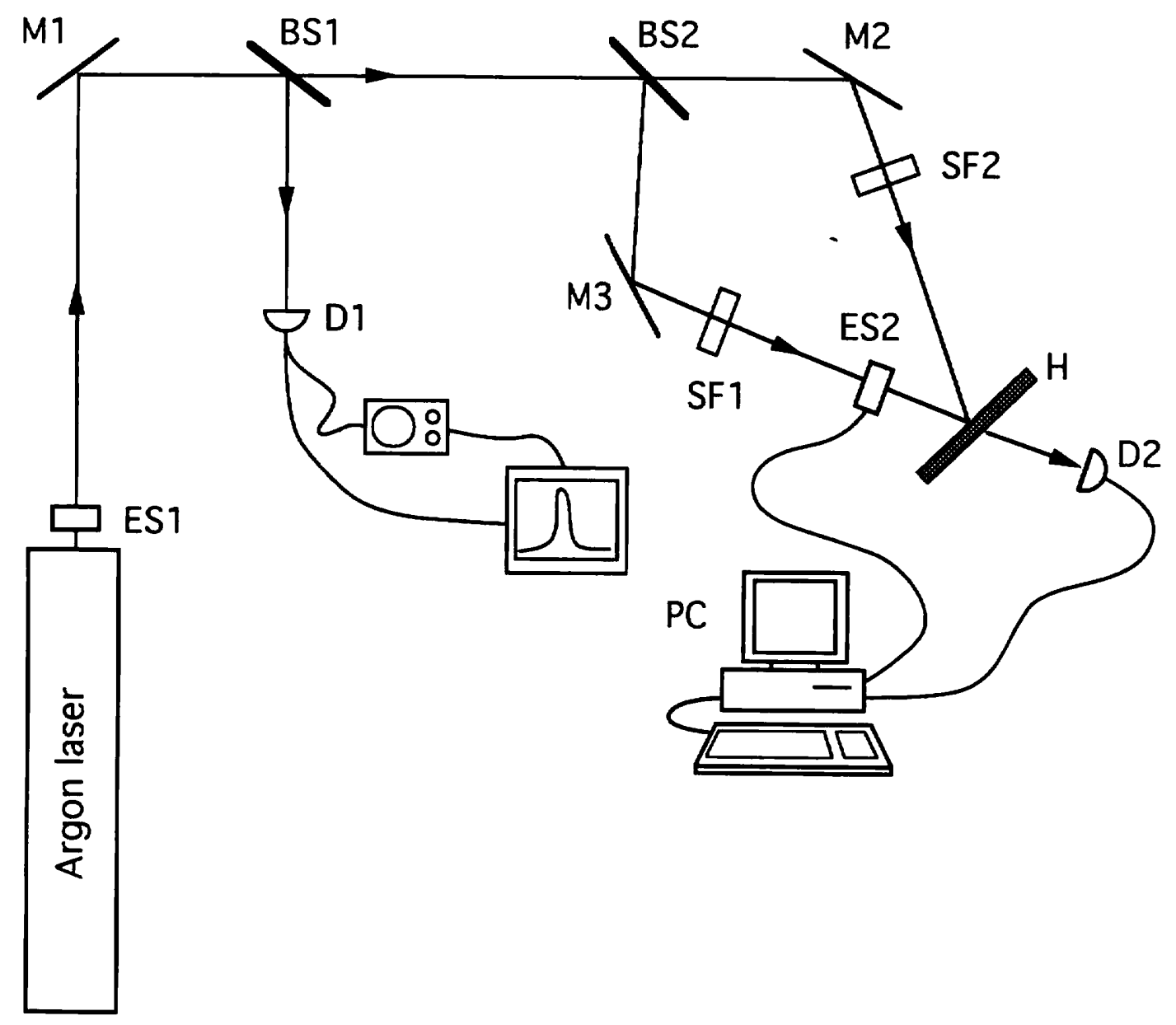

FIGURE 1 


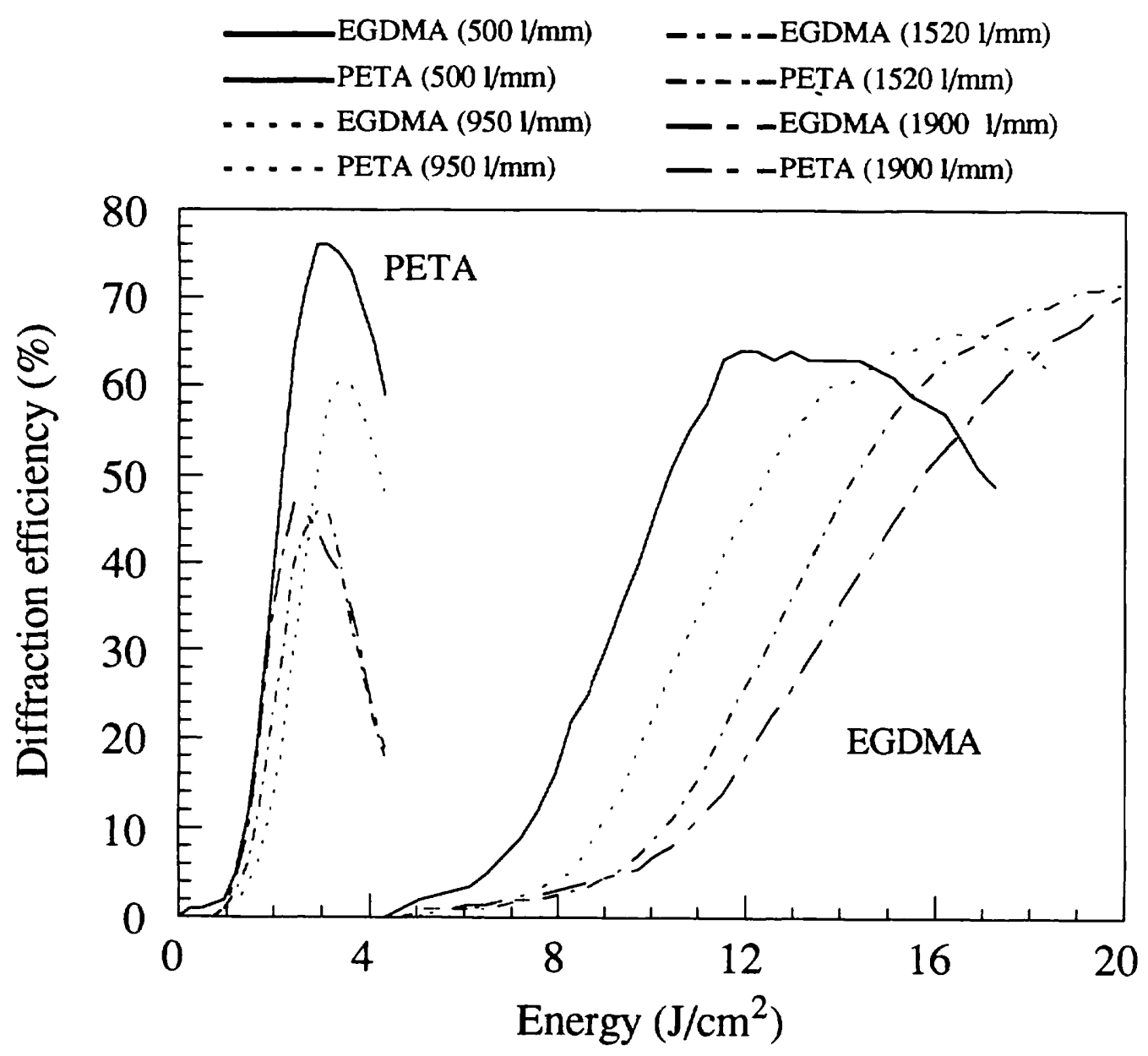


FIMIA GIL, Antonio, et al. "New photopolymer with trifunctional monomer for holographic applications". Applied Physics B: Lasers and Optics, Vol. 63, No. 2, pp. 151-153 (1996). ISSN 0946-2171. DOI: 10.1007/BF01095266

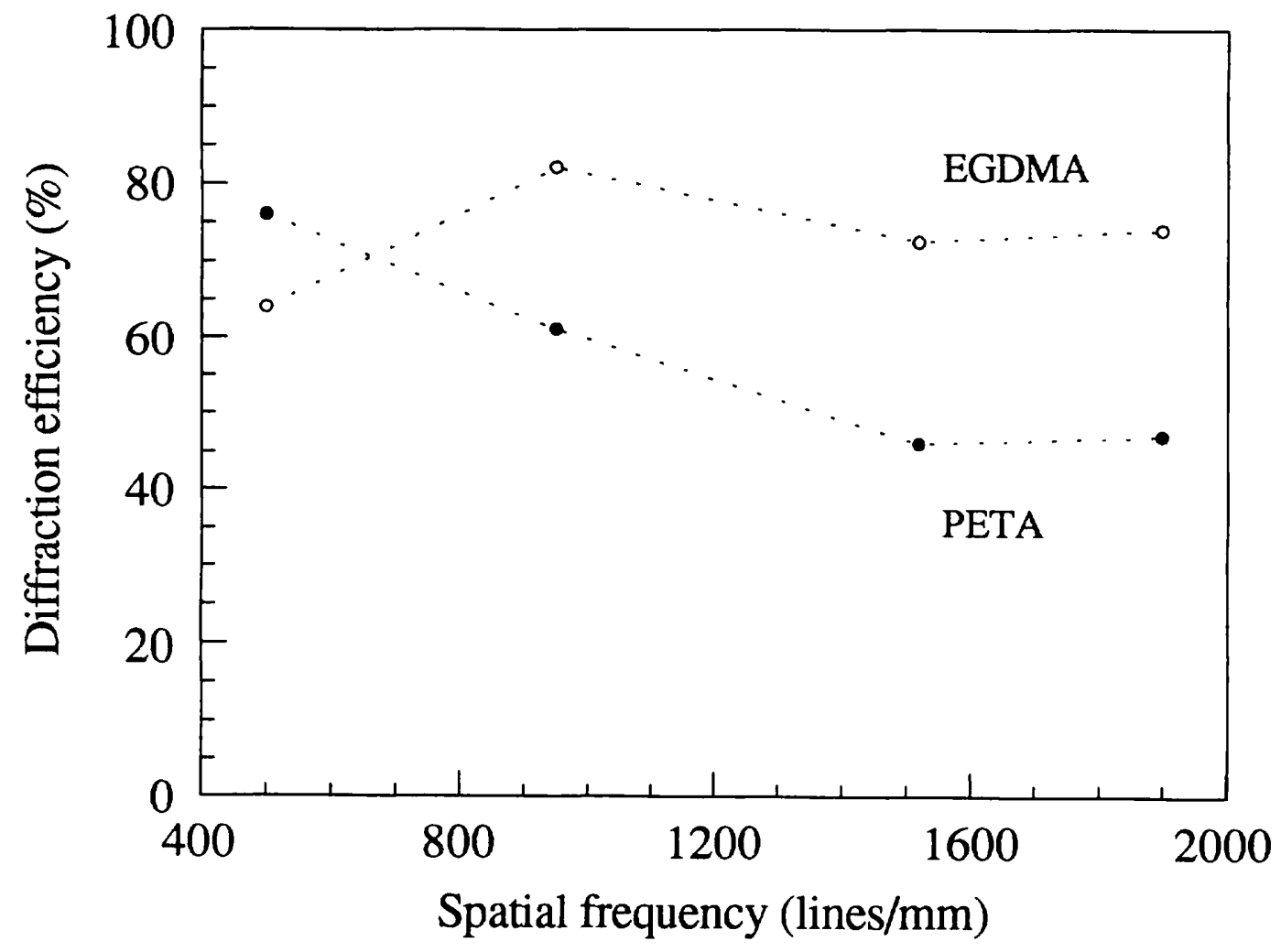

FiGURE 3
A.Fimia et al. 
FIMIA GIL, Antonio, et al. "New photopolymer with trifunctional monomer for holographic applications". Applied Physics B: Lasers and Optics, Vol. 63, No. 2, pp. 151-153 (1996). ISSN 0946-2171. DOI: 10.1007/BF01095266

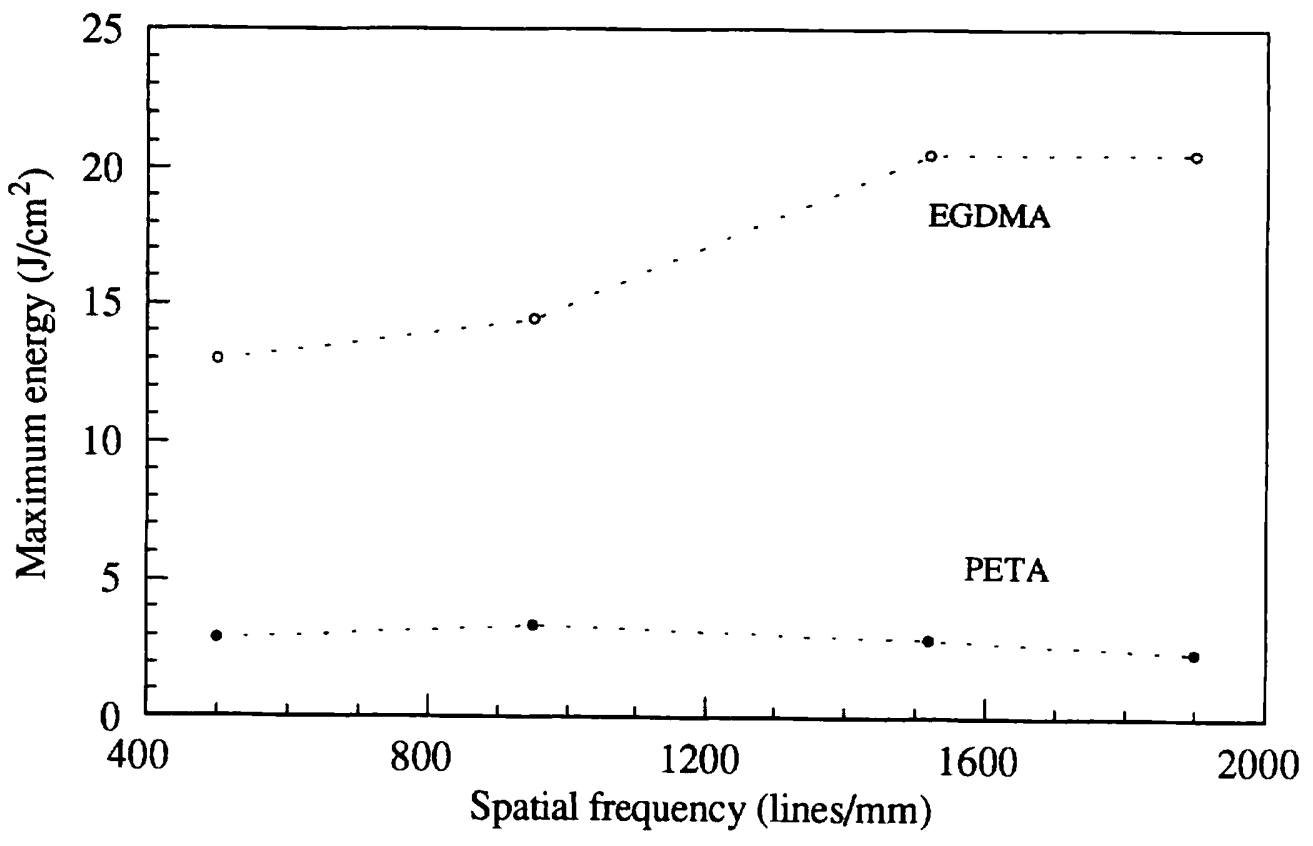

FIGURE 4 
FIMIA GIL, Antonio, et al. "New photopolymer with trifunctional monomer for holographic applications". Applied Physics B: Lasers and Optics, Vol. 63, No. 2, pp. 151-153 (1996). ISSN 0946-2171. DOI: 10.1007/BF01095266

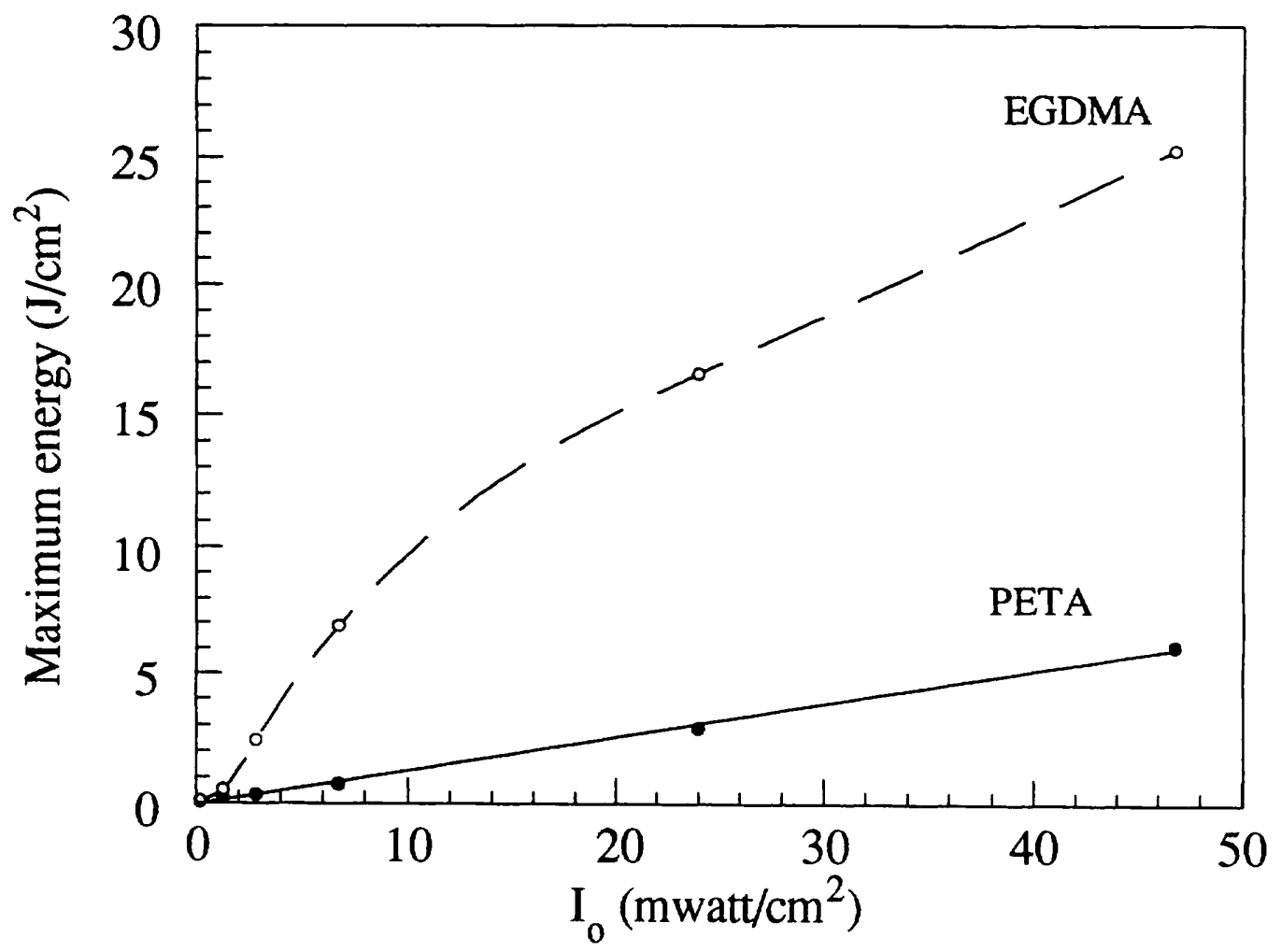

FIGURE 5

A. Finvia et al. 
FIMIA GIL, Antonio, et al. "New photopolymer with trifunctional monomer for holographic applications". Applied Physics B: Lasers and Optics, Vol. 63, No. 2, pp. 151-153 (1996). ISSN 0946-2171. DOI: 10.1007/BF01095266

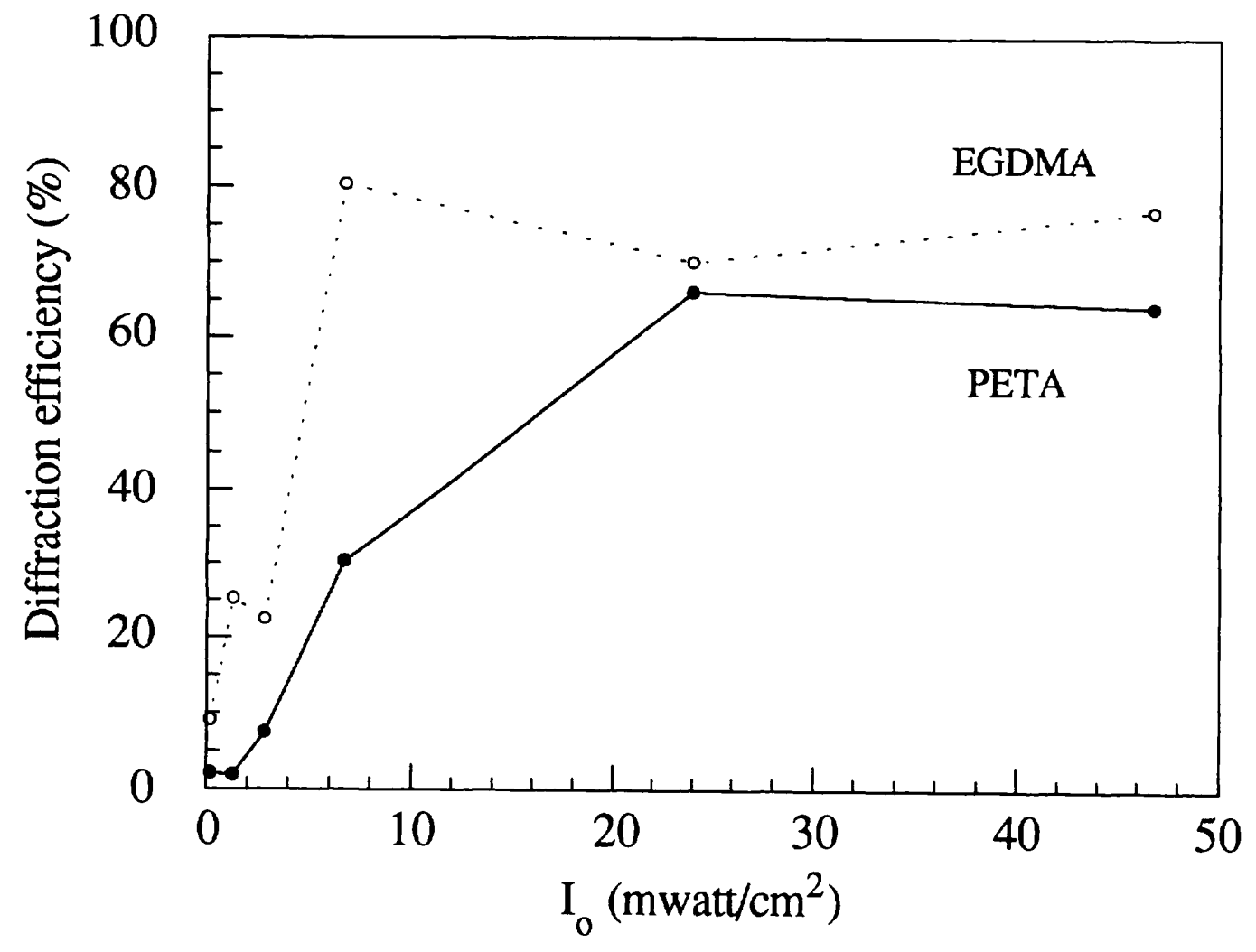

FIGURE 6

A. Fimia et al. 
FIMIA GIL, Antonio, et al. "New photopolymer with trifunctional monomer for holographic applications". Applied Physics B: Lasers and Optics, Vol. 63, No. 2, pp. 151-153 (1996). ISSN 0946-2171. DOI: 10.1007/BF01095266

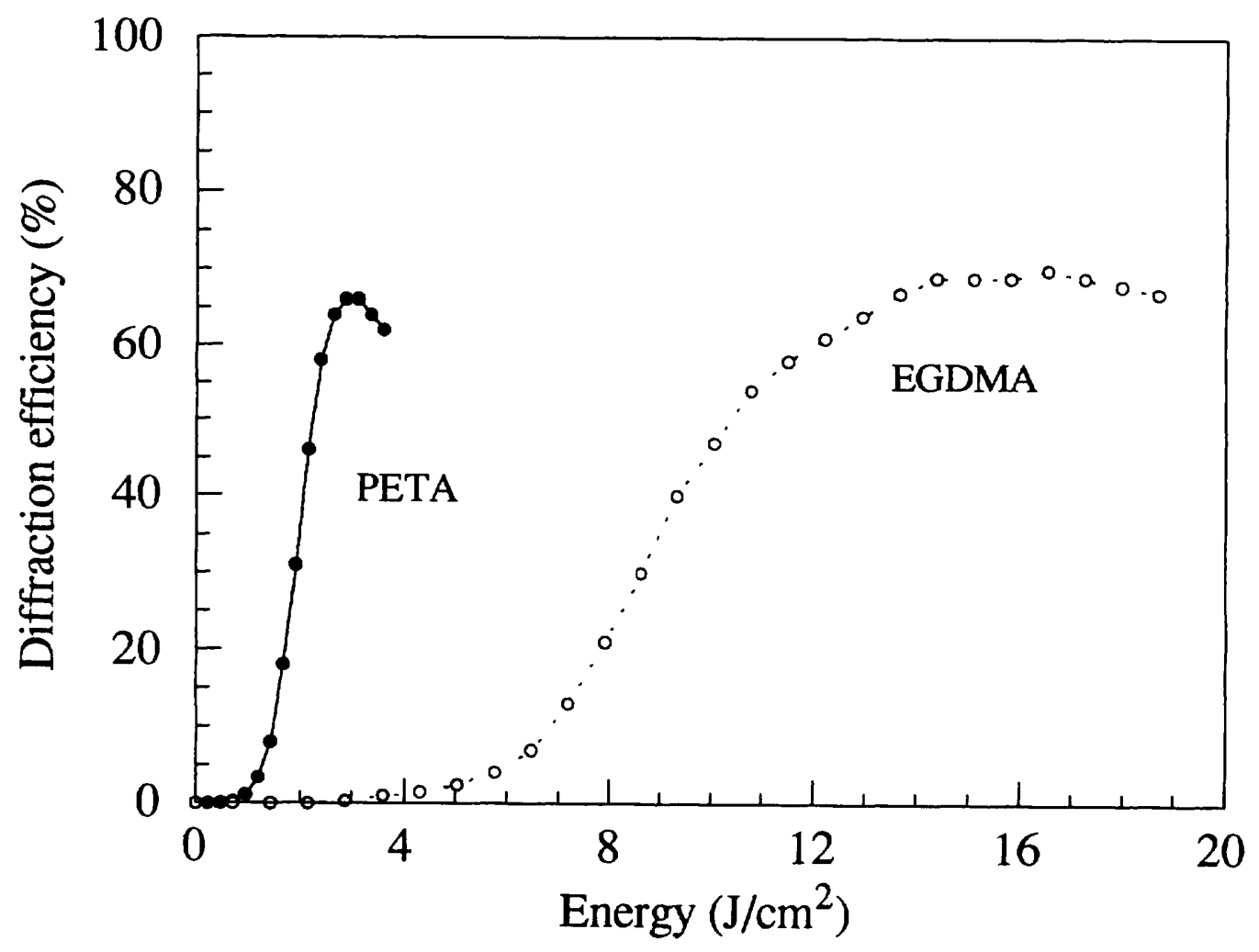

FIEURE 7 A.Fimia etal. 